TRANSACTIONS OF THE

AMERICAN MATHEMATICAL SOCIETY

Volume 349, Number 12, December 1997, Pages 5043-5071

S 0002-9947(97)01874-6

\title{
NON-ARCHIMEDEAN NEVANLINNA THEORY IN SEVERAL VARIABLES AND THE NON-ARCHIMEDEAN NEVANLINNA INVERSE PROBLEM
}

\author{
WILLIAM CHERRY AND ZHUAN YE
}

\begin{abstract}
Cartan's method is used to prove a several variable, non-Archimedean, Nevanlinna Second Main Theorem for hyperplanes in projective space. The corresponding defect relation is derived, but unlike in the complex case, we show that there can only be finitely many non-zero non-Archimedean defects. We then address the non-Archimedean Nevanlinna inverse problem, by showing that given a set of defects satisfying our conditions and a corresponding set of hyperplanes in projective space, there exists a non-Archimedean analytic function with the given defects at the specified hyperplanes, and with no other defects.
\end{abstract}

\section{History And Introduction}

Nevanlinna theory, broadly speaking, studies to what extent something like the Fundamental Theorem of Algebra holds for meromorphic functions. Unlike polynomials, transcendental meromorphic functions, in general, have infinitely many zeros. However, they have only finitely many zeros inside a disc of radius $r$. Therefore, in order to study the values of a meromorphic function, Nevanlinna theory associates to each meromorphic function $f$, three functions of $r$, the distance from the origin (for their precise definitions, see Nevanlinna [Ne 2]). The "characteristic" or "height" function $T_{f}(r)$ measures the growth of $f$ and should be thought of as the analogue of the degree of a polynomial. The "counting" function $N_{f}(a, r)$ counts the number of times (as a logarithmic average) $f$ takes on the value $a$ in the disc of radius $r$. Finally, the "mean-proximity function" $m_{f}(a, r)$ measures how often, on average, $f$ stays "close to" the value $a$ on the circle of radius $r$. Nevanlinna proved two "main" theorems about these functions. The so-called "First Main Theorem" states that

$$
T_{f}(r)=m_{f}(a, r)+N_{f}(a, r)+O(1)
$$

where the bounded term $O(1)$ depends on $f$ and $a$ but not on $r$. This should be thought of as a substitute for the Fundamental Theorem of Algebra in the following sense. The First Main Theorem says that $m_{f}(a, r)+N_{f}(a, r)$ is essentially independent of the value $a$, and this is analogous to the fact that a polynomial takes on every finite value the same number of times counting multiplicity. The First

Received by the editors October 14, 1995 and, in revised form, June 17, 1996.

1991 Mathematics Subject Classification. Primary 11J99, 11S80, 30D35, 32H30, 32 P05.

Key words and phrases. Non-Archimedean, Nevanlinna theory, p-adic, inverse problem, defect relations, projective space.

Financial support for the first author was provided in part by NSF grant \# DMS-9505041.

(C)1997 American Mathematical Society 
Main Theorem is weaker, though, in the sense that $f$ need not take on a value $a$ very often so long as it stays "close" to the value $a$ often. For example, the function $e^{z}$ never takes on the value 0 , but it is close to zero on about half of each circle of radius $r$. The function $e^{z}$ takes on all non-zero finite values $a$ equally often, but on any given large circle, it is "near" any non-zero value $a$ on only a very small arc.

Nevanlinna's Second Main Theorem is a deeper result which says in a very precise way that for most values of $a$, the counting function dominates the proximity function. More precisely (see [Ye 2] for this formulation),

Theorem 1.1. Nevanlinna's Second Main Theorem. Let $\phi(r)$ be an increasing function of $r$ such that

$$
\int_{1}^{\infty} \frac{1}{r \phi(r)} d r<\infty
$$

Let $a_{1}, \ldots, a_{q}$ be $q$ distinct points in $\mathbf{P}^{1}=\mathbf{C} \cup\{\infty\}$. Let $f$ be a non-rational meromorphic function. Assume for simplicity that $f(0) \neq 0, \infty, a_{j}$ and that $f^{\prime}(0) \neq 0$. Then

$$
(q-2) T_{f}(r)-\sum_{j=1}^{q} N_{f}\left(a_{j}, r\right)+N_{f, \operatorname{Ram}}(r) \leq \log T_{f}(r)+\log \phi\left(T_{f}(r)\right)+O(1),
$$

for all $r$ outside a set of finite Lebesgue measure.

The right hand side of the above inequality is called the "error term." The point is to take $\phi$ as small as possible, subject to the integral condition, although the measure of the set of values of $r$ for which the inequality does not hold grows as $\phi$ gets smaller. Classically, Nevanlinna took $\phi$ to be $(\log r)^{1+\varepsilon}$. For small $\phi$, the error term is small compared with $T_{f}$. The term $N_{f, \operatorname{Ram}}(r)$ counts how many times $f$ is ramified inside the disc of radius $r$. The assumptions on $f(0)$ and $f^{\prime}(0)$ are not essential, but they make the inequality easier to state. For examples illustrating the sharpness of the error term in Theorem 1.1, see [Ye 1].

As noted above, the Second Main Theorem tells us that for "most" values of $a, N_{f}(a, r)$ dominates the term $m_{f}(a, r)$. This motivates the definition of a "deficiency" or a "defect." We define the "defect" of a value $a$ to be

$$
\delta_{f}(a)=\liminf _{r \rightarrow \infty}\left\{1-\frac{N_{f}(a, r)}{T_{f}(r)}\right\} .
$$

A value $a$ is called "deficient" for $f$ if $\delta_{f}(a)>0$. Dividing the inequality in the Second Main Theorem by $T_{f}$ immediately gives the so-called "defect relation:"

$$
\sum_{a \in \mathbf{P}^{1}} \delta_{f}(a) \leq 2 .
$$

In [Ne 1], Nevanlinna asks whether this is the only restriction on defects, and proves some important special cases that suggest that it is. More precisely, Nevanlinna asked:

Question 1.2. Nevanlinna Inverse Problem. Let $\delta_{j}$ be a countable collection of numbers $0<\delta_{j} \leq 1$ such that

$$
\sum_{j} \delta_{j} \leq 2
$$


Let $\left\{a_{j}\right\}$ be distinct points in $\mathbf{P}^{1}$. Does there exist a meromorphic function $f$ such that $\delta_{f}\left(a_{j}\right)=\delta_{j}$ and $\delta_{f}(a)=0$ for all $a \notin\left\{a_{j}\right\}$ ?

Question 1.2 was answered in the affirmative by Drasin [Dr]. In fact, Drasin showed more. His theorem also included the so-called "ramification defects" coming from the ramification term in the Second Main Theorem, and he shows that one can find a meromorphic function $f$ solving the inverse problem of arbitrarily "small" infinite order.

Nevanlinna's theory of meromorphic functions was generalized by Cartan [Ca], and later independently by Ahlfors [Ah], to holomorphic curves in projective space. Kneser $[\mathrm{Kn}]$ proved the First Main Theorem for meromorphic functions of several variables, and much later, Carlson, Griffiths, Cowen, King, Stoll and others (see for instance, [C-G], [G-K], [L-C], [St], [Wo], [W-S], [Ye 2]) generalized Nevanlinna theory to several variables.

Motivated, at least in part, by the analogy Osgood [Os 1], [Os 2] and Vojta [Vo] make between Nevanlinna theory and Diophantine approximation, the study of Nevanlinna theory over non-Archimedean fields has been around since at least the early eighties. See [Bo 1], [Bo 2], [Ch 1], [Co 1], [Co 2], [Kh 1], and [Kh 2]. See also the 1971 paper of Adams and Straus [A-S], which uses the techniques, but not the language, of non-Archimedean Nevanlinna theory. Most of the papers cited above include proofs of analogous First and Second Main Theorems. For example, we have the following non-Archimedean Second Main Theorem:

Theorem 1.3. Non-Archimedean Second Main Theorem. Let $\mathbf{F}$ be an algebraically closed field of characteristic zero complete with respect to a non-trivial non-Archimedean valuation. Let $a_{1}, \ldots, a_{q}$ be $q$ distinct points in $\mathbf{P}^{1}(\mathbf{F})$. Let $f$ be a meromorphic function on $\mathbf{F}$. Then,

$$
(q-2) T_{f}(r)-\sum_{j=1}^{q} N_{f}\left(a_{j}, r\right)+N_{f, \operatorname{Ram}}(r) \leq-\log r+O(1)
$$

for all $r \geq 1$, where the constant $O(1)$ depends only on $q, a_{1}, \ldots, a_{q}$, and $f$. The dependence on $f$ can be removed if $f$ is normalized so that $f(0)=1$.

Notice that, unlike in the complex case, the error term in the non-Archimedean Second Main Theorem is bounded as $r \rightarrow \infty$.

As in the complex case, dividing both sides of the above inequality by $T_{f}$ gives us:

\section{Corollary 1.4. Defect Relation.}

$$
\sum_{a \in \mathbf{P}^{1}(\mathbf{F})} \delta_{f}(a) \leq 2 .
$$

As far as we know, no one has yet commented on the sharpness of these inequalities or investigated the Nevanlinna Inverse Problem in the non-Archimedean case. In this paper, we give an easy example to show that the non-Archimedean Second Main Theorem with the ramification term is sharp. However, we will show that for every meromorphic function there can be at most one deficient value. Thus, from the value-distribution theory point of view, non-Archimedean meromorphic functions behave more like ordinary polynomials than they do like meromorphic 
functions on the complex plane. This can be viewed as supporting evidence for the conjecture in [Ch 1] (see also [Ch 2]) that projective algebraic varieties contain non-trivial non-Archimedean analytic curves if and only if they contain rational curves.

Boutabaa [Bo 1], Cherry [Ch 1], and Khoái-Tu [K-T] have independently verified that Cartan's method of studying holomorphic curves in projective space generalizes to the one variable non-Archimedean case, and Khoái [Kh 3] began to study several variable non-Archimedean Nevanlinna theory. Ye [Ye 2], generalizing work of Vitter [Vi], shows that Cartan's method can prove sharp several variable hyperplane second main theorems for maps from $\mathbf{C}^{m}$ into projective space. In this paper, we show that Lang's [La] presentation of Cartan's method together with some key ideas in [Ye 2] can give the analogous non-Archimedean several variable hyperplane second main theorems. We also investigate the sharpness and inverse problems for the non-Archimedean curve and several variable cases. Again, we can see from the discussion of this paper that the non-Archimedean situation is easier and less interesting than the complex case, because the non-Archimedean maps behave very much like polynomial mappings.

When the first draft of this paper was submitted for publication, no proof of a non-Archimedean hyperplane second main theorem was readily available in the literature. While the first draft of this paper was with the referee, the work of Khoái-Tu $[\mathrm{K}-\mathrm{T}]$ appeared in print. Khoái-Tu made a significant contribution by recognizing the importance of an extra term neglected by Cherry [Ch 1] and Boutabaa [Bo 3], although present in [Bo 1]. We have incorporated this previously neglected term into the present paper, and we will say more about this at the appropriate place. The paper $[\mathrm{K}-\mathrm{T}]$ also includes several interesting applications of the hyperplane second main theorem, which we do not discuss here. The original content of the present paper is Lemma 2.3, which is the key to extending the proof to several variables, and section 6 , where defects and the inverse problem are discussed.

The authors would like to thank David Drasin for his comments and suggestions. The authors would also like to express their gratitude to the referee for many helpful comments and suggestions.

\section{Definitions, Notation, and Preliminary Results}

Throughout this paper, $\mathbf{F}$ will denote an algebraically closed field of characteristic 0 complete with respect to a non-trivial non-Archimedean valuation $v$. A non-Archimedean valuation $v$ is a real-valued function on the non-zero elements of $\mathbf{F}$ such that if $z$ and $w$ are non-zero elements of $\mathbf{F}$, then $v(z w)=v(z)+v(w)$, and $v(z+w) \geq \min \{v(z), v(w)\}$. The valuation $v$ is said to be "non-trivial" if there exists some non-zero element $z$ in $\mathbf{F}$ such that $v(z) \neq 0$. Given a positive real number $\pi$, we define a non-Archimedean absolute value ||$_{v}$ on $\mathbf{F}$ by

$$
|z|_{v}=\pi^{-v(z)} \text {. }
$$

Note that ||$_{v}$ satisfies the non-Archimedean triangle inequality

$$
|z+w|_{v} \leq \max \left\{|z|_{v},|w|_{v}\right\}
$$

and we will use this property over and over again without comment in the sequel. The most important example of such a field $\mathbf{F}$ is the field $\mathbf{C}_{p}$ of $p$-adic complex 
numbers. To get $\mathbf{C}_{p}$, let $p$ be a prime number and start by defining $v$ on $\mathbf{Q}$, the field of rational numbers. For any rational number $q$, write $q=p^{n} \frac{a}{b}$, where $n$ is an integer and $p$ does not divide $a$ or $b$. Define $v(q)=n$. This is a non-Archimedean valuation on $\mathbf{Q}$, and it is customary to normalize the absolute value so that

$$
|q|_{v}=p^{-v(q)},
$$

or in other words, so that $\pi=p$. The rational number field $\mathbf{Q}$ can be completed with respect to this absolute value to get the field of $p$-adic numbers, denoted $\mathbf{Q}_{p}$. The valuation $v$ on $\mathbf{Q}$ (and hence $\mathbf{Q}_{p}$ ) can be extended to the algebraic closure of $\mathbf{Q}_{p}$. The field $\mathbf{C}_{p}$ is the completion of the algebraic closure of $\mathbf{Q}_{p}$ under this valuation. Note that it is a theorem that $\mathbf{C}_{p}$ is algebraically closed.

Let $\mathbf{F} \leq$ be the set of elements $z$ of $\mathbf{F}$ such that $|z|_{v} \leq 1$. Let $\mathbf{F}^{<}$be the set of elements $z$ of $\mathbf{F}$ such that $|z|_{v}<1$. Define two elements $z_{1}$ and $z_{2}$ of $\mathbf{F} \leq$ to be equivalent if $z_{1}-z_{2}$ is in $\mathbf{F}^{<}$. Denote the equivalence class of an element $z$ by $\tilde{z}$. The set of all such equivalence classes forms an algebraically closed field, called the residue class field, and will be denoted by $\widetilde{\mathbf{F}}$.

Let $\mathbf{F}^{m}$ be the $m$-th Cartesian product of $\mathbf{F}$. We let $\mathbf{B}^{m}(r)$ be the "closed" ball of radius $r$ in $\mathbf{F}^{m}$, which is defined by

$$
\mathbf{B}^{m}(r)=\left\{\left(z_{1}, \ldots, z_{m}\right) \in \mathbf{F}^{m}:\left|z_{j}\right|_{v} \leq r \text { for } j=1, \ldots m\right\} .
$$

Note that $\mathbf{B}^{m}(r)$ is actually both open and closed, but we use the adjective "closed" because of the $\leq$ in the definition. Also note that $\mathbf{B}^{m}(r)$ is more analogous to a polydisc than to a ball, but it seems customary to refer to it as a ball.

If $z_{1}, \ldots, z_{m}$ are $\mathbf{F}$-valued variables, we use $z$ to denote the $m$-tuple $\left(z_{1}, \ldots, z_{m}\right)$. We use multi-index notation, so if $\gamma=\left(\gamma_{1}, \ldots, \gamma_{m}\right)$, where the $\gamma_{j}$ are non-negative integers, then by definition

$$
z^{\gamma}=z_{1}^{\gamma_{1}} \cdots z_{m}^{\gamma_{m}}, \quad|\gamma|=\gamma_{1}+\cdots+\gamma_{m}, \quad \text { and } \quad \gamma !=\gamma_{1} ! \cdots \gamma_{m} ! .
$$

By an analytic function $f$ on $\mathbf{B}^{m}(r)$ we mean a formal power series

$$
\sum_{\gamma} a_{\gamma} z^{\gamma}
$$

in $m$ variables, $z_{1}, \ldots, z_{m}$, with coefficients in $\mathbf{F}$ such that

$$
\lim _{|\gamma| \rightarrow \infty}\left|a_{\gamma}\right|_{v} r^{|\gamma|}=0 .
$$

If

$$
\lim _{|\gamma| \rightarrow \infty}\left|a_{\gamma}\right|_{v} r^{|\gamma|}=0
$$

for all non-negative real numbers $r$, then $f$ can be considered to be an analytic function on all of $\mathbf{F}^{m}$.

If

$$
f=\sum_{\gamma} a_{\gamma} z^{\gamma}
$$

is analytic on $\mathbf{B}^{m}(r)$, then define

$$
|f|_{r}=\sup _{\gamma}\left|a_{\gamma}\right|_{v} r^{|\gamma|}
$$


Note that ||$_{r}$ is multiplicative, meaning that if $f$ and $g$ are analytic, then

$$
|f g|_{r}=|f|_{r}|g|_{r} \text {. }
$$

We remark that if we have a sequence of functions $f_{n}$, analytic on balls $\mathbf{B}^{m}\left(r_{n}\right)$, and if for every $r>0$ and every $\varepsilon>0$ there exists an integer $N$ so that whenever $m, n \geq N$ we have $r_{n}, r_{m} \geq r$ and $\left|f_{n}-f_{m}\right|_{r}<\varepsilon$, then $f_{n} \rightarrow f$, where $f$ is an analytic function on $\mathbf{F}^{m}$.

We will have occasion to use infinite products, so we state a proposition about their convergence.

Proposition 2.1. Let $f_{n}$ be analytic functions on $\mathbf{B}^{m}(r)$ such that

$$
\lim _{n \rightarrow \infty}\left|1-f_{n}\right|_{r}=0
$$

Then

$$
f=\prod_{n=1}^{\infty} f_{n}
$$

is an analytic function on $\mathbf{B}^{m}(r)$. If the hypotheses hold for all $r>0$, then $f$ is analytic on $\mathbf{F}^{m}$.

Proof. Consider the partial products,

$$
P_{N}=\prod_{n=1}^{N} f_{n}
$$

We need to check that $\left|P_{N}-P_{M}\right|_{r}$ tends to zero as $\min \{N, M\} \rightarrow \infty$. Without loss of generality, assume that $N \geq M$. Then,

$$
\left|P_{M}-P_{N}\right|_{r}=\left.\left|\prod_{n=1}^{M} f_{n}\right|\right|_{r}\left|1-\prod_{n=M+1}^{N} f_{n}\right|_{r}
$$

Because $\left|1-f_{n}\right|_{r} \rightarrow 0$, there exists an $N_{0}$ such that for all $n \geq N_{0},\left|f_{n}\right|_{r}=1$. Therefore for $M \geq N_{0}$,

$$
\left|\prod_{n=1}^{M} f_{n}\right|_{r}=\left|\prod_{n=1}^{N_{0}} f_{n}\right|_{r}
$$

On the other hand, if $M \geq N_{0}$, then

$$
\left|1-\prod_{n=M+1}^{N} f_{n}\right|_{r}=\left|1-\prod_{n=M+1}^{N}\left(1-\left(1-f_{n}\right)\right)\right| \leq \sup _{n>M}\left|1-f_{n}\right|_{r}
$$

The right hand side tends to zero as $M \rightarrow \infty$ by assumption, and the proposition follows.

We say that an analytic function $g$ divides an analytic function $f$ if $f=g h$ for some analytic function $h$, and we say that $g$ is a greatest common divisor of $n$ analytic functions $f_{1}, \ldots, f_{n}$ if whenever an analytic function $h$ divides each of the non-zero $f_{i}$ then $h$ also divides $g$. We say that $n$ analytic functions $f_{1}, \ldots, f_{n}$ are without common factors if 1 is a greatest common divisor. 
By a meromorphic function $f$ on $\mathbf{F}^{m}$ (or on $\mathbf{B}^{m}(r)$ ) we will mean the quotient of two analytic functions $f_{1} / f_{2}$ such that $f_{1}$ and $f_{2}$ do not have any common factors in the ring of analytic functions on $\mathbf{F}^{m}$ (or on $\mathbf{B}^{m}(r)$ ). Note that for convenience, we consider the constant function $\infty$ to be a meromorphic function, and this is slightly non-standard.

Given any two analytic functions $f_{1}$ and $f_{2}$, possibly with common factors, one could ask whether $f_{1} / f_{2}$ makes sense as a meromorphic function. This is clearly equivalent to asking if greatest common divisors exist in the ring of analytic functions on $\mathbf{F}^{m}$ (or on $\mathbf{B}^{m}(r)$ ). If there exists $a$ in $\mathbf{F}$ with $|a|_{v}=r$, then the ring of analytic functions on $\mathbf{B}^{m}(r)$ is a unique factorization domain (see [BGR], Theorem 5.2.6/1), and so greatest common divisors exist. We know of no reference in the literature for the existence of greatest common divisors in the ring of analytic functions on $\mathbf{F}^{m}$, so for the sake of completeness we have included an appendix containing an argument of W. Lütkebohmert [Lü] for the existence of greatest common divisors in that ring.

Note that because ||$_{r}$ is multiplicative on analytic functions, we can uniquely extend ||$_{r}$ to meromorphic functions $f=g / h$, where $g$ and $h$ are analytic, by defining

$$
|f|_{r}=\frac{|g|_{r}}{|h|_{r}}
$$

The mean-proximity function measures how close a function is to a certain value on the boundary of the ball of radius $r$. The mean-proximity function $m_{f}(\infty, r)$ should measure how close the meromorphic function $f$ stays to infinity on the boundary of the ball of radius $r$. Define

$$
m_{f}(\infty, r)=\max \left\{0, \log _{\pi}|f|_{r}\right\} .
$$

If $a$ is an element of $\mathbf{F}$, then $f$ is close to $a$ whenever $1 /(f-a)$ is large, so define

$$
m_{f}(a, r)=m_{1 /(f-a)}(\infty, r) .
$$

To define the counting function for analytic or meromorphic functions of several variables is a little more subtle. The counting function counts, as a logarithmic average, how many times a function takes on a particular value in a disc. It is clear what to do in the one variable case, so for the several variable case we restrict to a generic line through the origin. The following two results are what allow us to do this. We begin by recalling the non-Archimedean Weierstrass Preparation Theorem in one variable:

Theorem 2.2. Weierstrass Preparation Theorem. Let

$$
f=\sum_{j=0}^{\infty} a_{j} z^{j}
$$

be an analytic function on $\mathbf{B}^{1}(r)$. Assume that $f$ is not identically zero. Let $d$ be the largest integer such that

$$
\left|a_{d}\right|_{v} r^{d}=\sup _{j}\left|a_{j}\right|_{v} r^{j}
$$


Then, there exists a unique monic polynomial $P$ of degree $d$ and a function $g$ which is analytic on $\mathbf{B}^{1}(r)$ such that $f=P g$. Furthermore, $g$ does not have any zeros inside $\mathbf{B}^{1}(r)$, and $P$ has exactly d zeros, counting multiplicity, on $\mathbf{B}^{1}(r)$.

The existence of $P$ and $g$ follows from Hensel's Lemma and the continuity of Euclid's algorithm. The statement about the zeros follows from Newton polygons. See $[\mathrm{BGR}]$ and $[\mathrm{Am}]$ for complete proofs.

Next, the following technical lemma tells us we can use the Weierstrass Preparation Theorem to count zeros by slicing with a generic line through the origin.

Lemma 2.3. Let $r$ be a positive real number, and let

$$
f\left(z_{1}, \ldots, z_{m}\right)=\sum_{\gamma} c_{\gamma} z^{\gamma}
$$

be an analytic function on $\mathbf{B}^{m}(r)$ in $\mathbf{F}^{m}$. Assume that $f$ is not identically zero. Let $u=\left(u_{1}, \ldots, u_{m}\right)$ be an $m$-tuple of elements in $\mathbf{F}$ with $\left|u_{j}\right|_{v}=1$ for all $j$, and let $f_{u}(z)=f\left(u_{1} z, \ldots, u_{m} z\right)$. If $f_{u}$ is not identically zero, then write $f_{u}=P_{u} g_{u}$, where $P_{u}$ and $g_{u}$ are the factors given by the Weierstrass Preparation Theorem (Theorem 2.2) applied to $f_{u}$ on $\mathbf{B}^{1}(r)$. Then, there exists a non-zero polynomial equation in $m$ variables with coefficients in $\widetilde{\mathbf{F}}$ such that if $\tilde{u}=\left(\tilde{u}_{1}, \ldots, \tilde{u}_{m}\right)$ is not a solution to the equation, then

$$
\sup \left\{|\gamma|:\left|c_{\gamma}\right|_{v} r^{|\gamma|}=|f|_{r}\right\}=\operatorname{deg} P_{u}=\text { number of zeros of } f_{u} \text { in } \mathbf{B}^{1}(r) .
$$

Proof. To begin, note that for any $u$,

$$
f_{u}(z)=\sum_{j=0}^{\infty} b_{j} z^{j},
$$

where

$$
b_{j}=\sum_{|\gamma|=j} c_{\gamma} u^{\gamma}
$$

Note that for each $j$,

$$
\left|b_{j}\right|_{v} \leq \sup _{|\gamma|=j}\left|c_{\gamma}\right|_{v}
$$

Hence,

$$
\left|f_{u}\right|_{r}=\sup _{j}\left|b_{j}\right|_{v} r^{j} \leq \sup _{j} \sup _{|\gamma|=j}\left|c_{\gamma}\right|_{v} r^{j}=|f|_{r} .
$$

Now, for each $j$ such that

$$
\sup _{|\gamma|=j}\left|c_{\gamma}\right|_{v} \neq 0
$$

let $a_{j}$ be an element of $\mathbf{F}$ so that

$$
\sup _{|\gamma|=j}\left|a_{j} c_{\gamma}\right|_{v}=1
$$

Let $\widetilde{P_{j}}$ be the homogeneous polynomial of degree $j$ with coefficients in $\widetilde{\mathbf{F}}$ defined by

$$
\widetilde{P_{j}}(\tilde{u})=\sum_{|\gamma|=j} \widetilde{a_{j} c_{\gamma}} \tilde{u}^{\gamma}
$$


The polynomial $\widetilde{P_{j}}$ is not identically zero, since for at least one multi-index $\gamma$ we have $\left|a_{j} c_{\gamma}\right|_{v}=1$. Furthermore, if $\tilde{u}$ is not a solution to $\widetilde{P_{j}}(\tilde{u})=0$, then we have equality in $(*)$.

Let $J=\sup \left\{|\gamma|:\left|c_{\gamma}\right|_{v} r^{|\gamma|}=|f|_{r}\right\}$. We want to show that $\widetilde{P_{J}}$ is the polynomial required by the lemma. Indeed, let $u$ be such that $\tilde{u}$ is not a solution of $\widetilde{P_{J}}(\tilde{u})=0$. As we observed above, this means that

$$
\left|b_{J}\right|_{v}=\sup _{|\gamma|=J}\left|c_{\gamma}\right|_{v}, \quad \text { and so } \quad\left|b_{J}\right|_{v} r^{J}=\sup _{|\gamma|=J}\left|c_{\gamma}\right|_{v} r^{J}=|f|_{r} .
$$

Hence, $\left|f_{u}\right|_{r}=|f|_{r}$. Moreover, by the choice of $J$, for all $j>J$, we have

$$
\left|b_{j}\right|_{v} r^{j} \leq \sup _{|\gamma|=j}\left|c_{\gamma}\right|_{v} r^{j}<|f|_{r}
$$

and so

$$
\sup \left\{j:\left|b_{j}\right|_{v} r^{j}=\left|f_{u}\right|_{r}\right\}=\sup \left\{|\gamma|:\left|c_{\gamma}\right|_{v} r^{\gamma}=|f|_{r}\right\}
$$

whereby the lemma follows.

We also recall here the following useful corollary.

Corollary 2.4. If $f$ is an analytic function on $\mathbf{F}^{m}$ that is never zero, then $f$ is constant.

We are now ready to define the counting function for analytic functions $f$. Let

$$
f=\sum_{\gamma} c_{\gamma} z^{\gamma}
$$

be analytic and not identically zero. Let $u=\left(u_{1}, \ldots, u_{m}\right)$ be an $m$-tuple of elements of $\mathbf{F}$ with $\left|u_{i}\right|_{v}=1$ for all $i$, and such that $\tilde{u}$ is generic in the sense that it does not satisfy the polynomial equation of Lemma 2.3. Define the unintegrated counting function $n_{f}(0, r)$ to be the number of zeros with absolute value $\leq r$ of the one variable function $f_{u}(z)=f\left(u_{1} z, \ldots, u_{m} z\right)$. Theorem 2.2 then tells us that

$$
n_{f}(0, r)=\sup \left\{|\gamma|:\left|c_{\gamma}\right|_{v} r^{\gamma}=|f|_{r}\right\},
$$

which allows us to easily compute $n_{f}(0, r)$. If $f=g / h$ is a meromorphic function and $g$ and $h$ do not have any common factors, then define the unintegrated counting function $n_{f}(\infty, r)$ by

$$
n_{f}(\infty, r)=n_{h}(0, r)
$$

For $a$ an element of $\mathbf{F}$ and $f$ a meromorphic function not identically equal to $a$, define

$$
n_{f}(a, r)=n_{1 /(f-a)}(\infty, r) .
$$

Define the order $\operatorname{ord}_{f}(r)$ of a meromorphic function which is not identically equal to zero or infinity, on the ball of radius $r$ to be

$$
\operatorname{ord}_{f}(r)=n_{f}(0, r)-n_{f}(\infty, r)
$$

Finally, for $a$ in $\mathbf{P}^{1}(\mathbf{F})$, and a meromorphic function $f$ not identically equal to $a$, define the integrated counting function $N_{f}(a, r)$ by

$$
N_{f}(a, r)=n_{f}(a, 0) \log _{\pi} r+\frac{1}{\ln \pi} \int_{0}^{r}\left(n_{f}(a, t)-n_{f}(a, 0)\right) \frac{d t}{t} .
$$


As an immediate consequence of Lemma 2.3 and Theorem 2.2 we have

Proposition 2.5. If $f$ is a meromorphic function on $\mathbf{F}^{m}$ that never takes on the value $a$ in $\mathbf{P}^{1}(\mathbf{F})$, then $N_{f}(a, r)=0$ for all $r$.

Finally, we define the height or characteristic function

$$
T_{f}(a, r)=m_{f}(a, r)+N_{f}(a, r) .
$$

Let $\mathbf{P}^{n}=\mathbf{P}^{n}(\mathbf{F})$ denote projective $n$-space over $\mathbf{F}$. By a meromorphic map $f: \mathbf{F}^{m} \rightarrow \mathbf{P}^{n}$, we mean an equivalence class of $(n+1)$-tuples of analytic functions $\left(f_{0}, \ldots, f_{n}\right)$ such that $f_{0}, \ldots, f_{n}$ do not have any common factors in the ring of analytic functions on $\mathbf{F}^{m}$ and such that not all of the $f_{j}$ are identically zero. Two $(n+1)$-tuples $\left(f_{0}, \ldots, f_{n}\right)$ and $\left(g_{0}, \ldots, g_{n}\right)$ are equivalent (or define the same meromorphic map to $\left.\mathbf{P}^{n}\right)$ if there exists a constant $c$ such that $f_{i}=c g_{i}$ for all $i$. (Recall from Corollary 2.4 that the only invertible analytic functions on $\mathbf{F}^{m}$ are the constants.) An $(n+1)$-tuple of meromorphic functions $\left(g_{0}, \ldots, g_{n}\right)$, not all identically zero, can be thought of as representing a meromorphic map from $\mathbf{F}^{m}$ to $\mathbf{P}^{n}$ by clearing denominators and pulling out common factors. (To know that we can pull out the common factor requires Proposition 7.1.) Unless otherwise noted, an $(n+1)$-tuple $\left(f_{0}, \ldots, f_{n}\right)$ representing a meromorphic map will consist of analytic functions without common factors.

In the next several paragraphs, we define the Nevanlinna functions for hyperplanes in $\mathbf{P}^{n}$. We want to point out that our definitions will depend on a choice of projective coordinates on $\mathbf{P}^{n}$, which is already implicit in our choosing coordinate functions $f_{0}, \ldots, f_{n}$, but we will see immediately following our definitions that a linear change in coordinates changes the Nevanlinna functions by at most a bounded term, and hence we suppress the dependence on coordinates from the notation.

Now then, let $x_{0}, \ldots, x_{n}$ be projective coordinates on $\mathbf{P}^{n}$, and let $H_{0}$ be the hyperplane defined by $x_{0}=0$. Let $f=\left(f_{0}, \ldots, f_{m}\right)$ be a meromorphic map from $\mathbf{F}^{m}$ to $\mathbf{P}^{n}$ such that the image of $f$ is not contained in $H_{0}$ (i.e. $f_{0}$ is not identically zero). We define the mean-proximity function $m_{f}\left(H_{0}, r\right)$ by

$$
m_{f}\left(H_{0}, r\right)=\max _{i} m_{f_{i} / f_{0}}(\infty, r) .
$$

Notice that if we replace $f_{i}$ by $c f_{i}$ for some non-zero constant $c$, then $m_{f}\left(H_{0}, r\right)$ does not change, so $m_{f}\left(H_{0}, r\right)$ is well-defined. If $H$ is any hyperplane in $\mathbf{P}^{n}$ not containing the image of a meromorphic map $f$, then let $\psi$ be an invertible projective linear transformation taking $H$ to $H_{0}$, and define $m_{f}(H, r)$ by

$$
m_{f}(H, r)=m_{\psi \circ f}\left(H_{0}, r\right) .
$$

We will see in a short while that $m_{f}(H, r)$ depends on $\psi$ only up to a bounded term.

To define the counting function, let $f=\left(f_{0}, \ldots, f_{m}\right)$ be a meromorphic map. Define $N_{f}\left(H_{0}, r\right)$ by

$$
N_{f}\left(H_{0}, r\right)=N_{f_{0}}(0, r) .
$$

This is well-defined because if the $f_{i}$ are without common factors, then $f_{0}$ is determined up to a multiplicative constant. For an arbitrary hyperplane $H$, let $\psi$ be an invertible linear transformation as above, and let

$$
N_{f}(H, r)=N_{\psi \circ f}\left(H_{0}, r\right) \text {. }
$$


Later, we will see that $N_{f}(H, r)$ is independent of the choice of $\psi$.

Finally, the height or characteristic function $T_{f}(H, r)$ is defined by

$$
T_{f}(H, r)=m_{f}(H, r)+N_{f}(H, r) .
$$

The following proposition helps us see that our definitions are, up to a bounded term, independent of coordinates on $\mathbf{P}^{n}$, but it will also be useful in other contexts.

Proposition 2.6. Let $f$ and $g$ be meromorphic functions on $\mathbf{F}^{m}$. Let a be a point in $\mathbf{P}^{1}$. Then,

$$
\begin{aligned}
m_{f+g}(\infty, r) & \leq \max \left\{m_{f}(\infty, r), m_{g}(\infty, r)\right\}, \\
m_{f g}(\infty, r) & \leq m_{f}(\infty, r)+m_{g}(\infty, r), \\
n_{f+g}(\infty, r) & \leq n_{f}(\infty, r)+n_{g}(\infty, r), \\
n_{f g}(\infty, r) & \leq n_{f}(\infty, r)+n_{g}(\infty, r), \\
N_{f+g}(\infty, r) & \leq N_{f}(\infty, r)+N_{g}(\infty, r), \\
N_{f g}(\infty, r) & \leq N_{f}(\infty, r)+N_{g}(\infty, r), \\
T_{f+g}(\infty, r) & \leq T_{f}(\infty, r)+T_{g}(\infty, r), \\
T_{f g}(\infty, r) & \leq T_{f}(\infty, r)+T_{g}(\infty, r) .
\end{aligned}
$$

Proof. Inequality (1a) follows from the fact that

$$
|f+g|_{r} \leq \max \left\{|f|_{r},|g|_{r}\right\} \text {. }
$$

Inequality (1b) follows from $|f g|_{r}=|f|_{r}|g|_{r}$. Inequalities (2a) and (2b) follow from the fact that a pole in a sum or a product can only come from a pole in one of the summands or factors. Inequalities (3a) and (3b) follow by integrating inequalities (2a) and (2b), and inequalities (4a) and (4b) follow by adding the inequalities in (1) and (3).

Proposition 2.7. Let $f$ be a meromorphic map from $\mathbf{F}^{m}$ to $\mathbf{P}^{n}$. Let $H$ be a hyperplane in $\mathbf{P}^{n}$. The counting function $N_{f}(H, r)$ is well-defined, and the other two Nevanlinna functions $m_{f}(H, r)$ and $T_{f}(H, r)$ are well-defined up to a bounded term.

Proof. It suffices to show that if $x_{0}, \ldots, x_{n}$ are projective coordinates on $\mathbf{P}^{n}$ and $\psi: \mathbf{P}^{n} \rightarrow \mathbf{P}^{n}$ is an invertible linear transformation that leaves the hyperplane $H_{0}$ given by $x_{0}=0$ fixed, then

$$
m_{f}\left(H_{0}, r\right)=m_{\psi \circ f}\left(H_{0}, r\right)+O(1) \quad \text { and } \quad N_{f}\left(H_{0}, r\right)=N_{\psi \circ f}\left(H_{0}, r\right) .
$$

Let $\left(f_{0}, \ldots, f_{n}\right)$ be coordinate representatives for $f$. We note that $\psi$ can be represented by an $(n+1) \times(n+1)$-matrix $\left(a_{i j}\right)$. Since $H_{0}$ is fixed, we may assume that $a_{0 j}=0$ for $j>0$, and since we are working projectively, we may scale things so that $\sup \left|a_{i j}\right|_{v}=1$. Then, by definition,

$$
m_{\psi \circ f}\left(H_{0}, r\right)=\max _{i} m_{g_{i} / g_{0}}(\infty, r), \quad \text { where } g_{i}=\sum_{j} a_{i j} f_{j} .
$$

By Proposition 2.6 and the fact that $\sup \left|a_{i j}\right|_{v}=1$,

$$
m_{g_{i} / g_{0}}(\infty, r) \leq \max _{j} m_{f_{j} / f_{0}}(\infty, r)+\max \left\{0, v\left(a_{00}\right)\right\} .
$$


Therefore,

$$
m_{\psi \circ f}\left(H_{0}, r\right) \leq m_{f}\left(H_{0}, r\right)+O(1) .
$$

Doing the same thing for $\psi^{-1}$ gives us

$$
m_{\psi \circ f}\left(H_{0}, r\right)=m_{f}\left(H_{0}, r\right)+O(1) .
$$

To show that $N_{f}\left(H_{0}, r\right)$ is well-defined, let $g_{i}$ be as above. Since the $f_{i}$ are analytic without common factors, so are the $g_{i}$. Indeed, if $h$ is a common factor of the $g_{i}$, then because $\psi$ is invertible, $h$ is also a common factor for the $f_{i}$, which are linear combinations of the $g_{i}$. Therefore,

$$
N_{\psi \circ f}\left(H_{0}, r\right)=N_{g_{0}}(0, r)=N_{a_{00} f_{0}}(0, r)=N_{f_{0}}(0, r)=N_{f}\left(H_{0}, r\right) .
$$

\section{Poisson-Jensen-Green Formula and the First Main Theorem}

The goal of this section is to prove the so-called "First Main Theorem," which tells us that the Nevanlinna height function $T_{f}(H, r)$, up to a bounded term, does not depend on the choice of the hyperplane $H$. The First Main Theorem is actually a corollary of the following non-Archimedean analogue to the Poisson-Jensen-Green formula in several complex variables. Before stating the result, we need some more notation; we need to quantify what happens to a meromorphic function $f$ at the origin. Let $f_{1}$ and $f_{2}$ be analytic functions such that $f=f_{1} / f_{2}$. Write

$$
f_{i}=\sum_{\gamma} c_{i, \gamma} z^{\gamma}, \quad i=1,2
$$

Let

$$
J_{i}=\inf \left\{|\gamma|: c_{i, \gamma} \neq 0\right\} .
$$

Define

$$
[f]_{0}=\frac{\sup _{|\gamma|=J_{1}}\left|c_{1, \gamma}\right|_{v}}{\sup _{|\gamma|=J_{2}}\left|c_{2, \gamma}\right|_{v}} .
$$

Clearly this is well-defined and multiplicative. For a meromorphic map $f$, the notation $[f]_{0}$ will refer to the collection of numbers $\left[f_{i} / f_{j}\right]_{0}$, where the $f_{i}$ are meromorphic coordinate functions representing $f$.

Theorem 3.1. Poisson-Jensen-Green Formula. Let $f$ be a meromorphic function on $\mathbf{B}^{m}(r)$. Then

$$
\log _{\pi}[f]_{0}=\log _{\pi}|f|_{r}-\frac{1}{\ln \pi} \int_{0}^{r}\left(\operatorname{ord}_{f}(t)-\operatorname{ord}_{f}(0)\right) \frac{d t}{t}-\operatorname{ord}_{f}(0) \log _{\pi} r
$$

Proof. Because $\log _{\pi}[f]_{0}, \log _{\pi}|f|_{r}$, and $\operatorname{ord}_{f}(r)$ are "multiplicative" in $f$, in the sense that they turn products into sums, it suffices to prove the theorem when $f$ is analytic. Write

$$
f=\sum_{\gamma} c_{\gamma} z^{\gamma}
$$


Let

$$
k_{0}=\inf \left\{|\gamma|: c_{\gamma} \neq 0\right\}
$$

For each $t<r$, let

$$
\begin{aligned}
k(t) & =\inf \left\{|\gamma|:\left|c_{\gamma}\right|_{v} t^{|\gamma|}=|f|_{t}\right\}, \\
K(t) & =\sup \left\{|\gamma|:\left|c_{\gamma}\right|_{v} t^{|\gamma|}=|f|_{t}\right\} .
\end{aligned}
$$

Call $t_{j}$ a critical point if $k\left(t_{j}\right) \neq K\left(t_{j}\right)$. We will prove the theorem by induction on the number of critical points $t_{j}<r$.

If there are no critical points $<r$, then $\operatorname{ord}_{f}(t)=\operatorname{ord}_{f}(0)=k_{0}$ for all $t<r$. Also,

$$
|f|_{r}=\sup _{|\gamma|=k_{0}}\left|c_{\gamma}\right|_{v} r^{k_{0}}=[f]_{0} r^{k_{0}},
$$

and the theorem is proved in this case.

Now assume there are $n$ critical points $t_{1}, \ldots, t_{n}$. By induction, we know that

$$
\log _{\pi}[f]_{0}=\log _{\pi}|f|_{t_{n}}-\frac{1}{\ln \pi} \int_{0}^{t_{n}}\left(\operatorname{ord}_{f}(t)-\operatorname{ord}_{f}(0)\right) \frac{d t}{t}-\operatorname{ord}_{f}(0) \log _{\pi} t_{n} .
$$

Furthermore,

$$
\begin{aligned}
\frac{1}{\ln \pi} \int_{t_{n}}^{r}\left(\operatorname{ord}_{f}(t)-\operatorname{ord}_{f}(0)\right) \frac{d t}{t} & +\operatorname{ord}_{f}(0)\left(\log _{\pi} r-\log _{\pi} t_{n}\right) \\
& =K\left(t_{n}\right)\left(\log _{\pi} r-\log _{\pi} t_{n}\right) .
\end{aligned}
$$

Since there are no critical points between $t_{n}$ and $r$,

$$
\begin{aligned}
\log _{\pi}|f|_{r} & =K\left(t_{n}\right) \log _{\pi} r+\sup _{|\gamma|=K\left(t_{n}\right)}\left\{\log _{\pi}\left|c_{\gamma}\right|_{v}\right\} \\
& =\log _{\pi}|f|_{t_{n}}+K\left(t_{n}\right)\left(\log _{\pi} r-\log _{\pi} t_{n}\right) .
\end{aligned}
$$

Hence the theorem follows by induction.

As a corollary, we get the so-called "First Main Theorem:"

Corollary 3.2. First Main Theorem. Let $f$ be a meromorphic map from $\mathbf{F}^{m}$ to $\mathbf{P}^{n}$. Let $H$ and $H^{\prime}$ be two hyperplanes in $\mathbf{P}^{n}$ such that the image of $f$ is not contained in either one. Then,

$$
T_{f}(H, r)=T_{f}\left(H^{\prime}, r\right)+O(1),
$$

where the $O(1)$ term depends on $H, H^{\prime}$, and $[f]_{0}$, but not on $r$.

Of course the theorem applies to the case $n=1$, so

Corollary 3.3. Let $f$ be a meromorphic function on $\mathbf{F}^{m}$. Let $a$ and $a^{\prime}$ be two points in $\mathbf{P}^{1}$. Then

$$
T_{f}(a, r)=T_{f}\left(a^{\prime}, r\right)+O(1)
$$


Proof of Corollary 3.2. By Proposition 2.7, $T_{f}(H, r)$ and $T_{f}\left(H^{\prime}, r\right)$ only change by a bounded term under an invertible linear change of coordinates, so we may choose coordinates $x_{0}, \ldots, x_{n}$ on $\mathbf{P}^{n}$ so that $H$ is given by $x_{0}=0$ and $H^{\prime}$ is given by $x_{1}=0$. Let $\left(f_{0}, \ldots, f_{n}\right)$ be analytic coordinate functions without common factors representing $f$. By definition,

$$
T_{f}(H, r)=\max _{i} \log _{\pi}\left|f_{i} / f_{0}\right|_{r}+N_{f_{0}}(0, r)
$$

and

$$
T_{f}\left(H^{\prime}, r\right)=\max _{i} \log _{\pi}\left|f_{i} / f_{1}\right|_{r}+N_{f_{1}}(0, r)
$$

Therefore,

$$
\begin{aligned}
T_{f}(H, r)-T_{f}\left(H^{\prime}, r\right)= & \max _{i} \log _{\pi}\left|f_{i} / f_{0}\right|_{r}-\max _{i} \log _{\pi}\left|f_{i} / f_{1}\right|_{r} \\
& -\left(N_{f_{1}}(0, r)-N_{f_{0}}(0, r)\right) \\
= & \log _{\pi}\left|f_{1} / f_{0}\right|_{r}-\left(N_{f_{1}}(0, r)-N_{f_{0}}(0, r)\right) \\
= & \log _{\pi}\left[f_{1} / f_{0}\right]_{0}=O(1),
\end{aligned}
$$

where the last line follows from Theorem 3.1.

In light of Corollary 3.2, from now on we omit the hyperplane $H$ from the notation involving the height, and just write $T_{f}(r)$.

Following Cartan $[\mathrm{Ca}]$, we note that the Poisson-Jensen-Green formula also gives us an alternative method for calculating $T_{f}(r)$. Namely,

Corollary 3.4. Let $f: \mathbf{F}^{m} \rightarrow \mathbf{P}^{n}$ be a meromorphic map. Let $\left(f_{0}, \ldots, f_{n}\right)$ be analytic coordinate representatives for $f$ without common factors. Then,

$$
T_{f}(r)=\max _{i} \log _{\pi}\left|f_{i}\right|_{r}+O(1)
$$

Proof. Trivially,

$$
\max _{i} \log _{\pi}\left|f_{i}\right|_{r}=\max _{i} \log _{\pi}\left|f_{i} / f_{0}\right|_{r}+\log _{\pi}\left|f_{0}\right|_{r} .
$$

Since $f_{0}$ is analytic, the definition of $N_{f_{0}}(0, r)$ and Theorem 3.1 imply

$$
\log _{\pi}\left|f_{0}\right|_{r}=N_{f_{0}}(0, r)+O(1)
$$

Since the $f_{i}$ are without common factors, $N_{f_{0}}(0, r)=N_{f}\left(H_{0}, r\right)$, so

$$
\begin{aligned}
\max _{i} \log _{\pi}\left|f_{i}\right|_{r} & =\max _{i} \log _{\pi}\left|f_{i} / f_{0}\right|_{r}+N_{f_{0}}(0, r)+O(1) \\
& =m_{f}\left(H_{0}, r\right)+N_{f}\left(H_{0}, r\right)+O(1)=T_{f}(r)+O(1) .
\end{aligned}
$$

We end this section with the following proposition, which tells us how we can use the height function $T_{f}(r)$ to tell whether or not $f$ is a rational function.

Proposition 3.5. Let $f$ be a meromorphic map from $\mathbf{F}^{m}$ to $\mathbf{P}^{n}$. Then

(i) $f$ is constant if and only if $T_{f}(r)=o\left(\log _{\pi} r\right)$.

(ii) $f$ is a rational map if and only if $T_{f}(r)=O\left(\log _{\pi} r\right)$.

In this case $\lim _{r \rightarrow \infty} T_{f}(r) / \log _{\pi} r$ equals the degree of $f$.

(iii) $f$ is non-constant if and only if there exist a constant $c$ in $\mathbf{R}$ and a number $A>0$ such that $T_{f}(r) \geq \log _{\pi} r+c$ for $r>A$. 
Proof. Our proof is modeled on that of Boutabaa [Bo 2]. Let $\left(f_{0}, \ldots, f_{n}\right)$ be analytic coordinate representatives without common factors for the mapping $f$. To show (ii), first suppose $T_{f}(r)=O\left(\log _{\pi} r\right)$. This implies that there is a constant $\alpha$ such that $T_{f}(r) \leq \alpha \log _{\pi} r$, for all $r$ sufficiently large. We want to show that the $f_{i}$ are polynomials. Let $H_{i}$ be one of the coordinate hyperplanes. Note that

$$
\begin{aligned}
N_{f}\left(H_{i}, r^{2}\right) & =n_{f_{i}}(0,0) \log _{\pi} r^{2}+\frac{1}{\ln \pi} \int_{0}^{r^{2}}\left(n_{f_{i}}(0, t)-n_{f_{i}}(0,0)\right) \frac{d t}{t} \\
& \geq n_{f_{i}}(0,0) \log _{\pi} r^{2}+\left(n_{f_{i}}(0, r)-n_{f_{i}}(0,0)\right) \log _{\pi} r \\
& \quad+\frac{1}{\ln \pi} \int_{0}^{r}\left(n_{f_{i}}(0, t)-n_{f_{i}}(0,0)\right) \frac{d t}{t} \\
& \geq n_{f_{i}}(0, r) \log _{\pi} r .
\end{aligned}
$$

Therefore

$$
n_{f_{i}}(0, r) \leq \frac{N_{f}\left(H_{j}, r^{2}\right)}{\log _{\pi} r} \leq \frac{T_{f}\left(r^{2}\right)+O(1)}{\log _{\pi} r} \leq \frac{\alpha \log _{\pi} r^{2}+O(1)}{\log _{\pi} r}=2 \alpha+o(1)
$$

for all $i$. Lemma 2.3 and Theorem 2.2 then easily imply that all the $f_{i}$ are polynomials.

Now assume that $f$ is a rational map. In this case we may choose coordinate functions $\left(P_{0}, \ldots, P_{n}\right)$ representing $f$ which are all polynomials without common factors. Let $d_{i}=\operatorname{deg} P_{i}$ and $D=\max d_{i}$. We want to show that

$$
\lim _{r \rightarrow \infty} \frac{T_{f}(r)}{\log _{\pi} r}=D .
$$

For $r \gg 0, \log _{\pi}\left|P_{i}\right|_{r}=d_{i} \log _{\pi} r$, and therefore by Corollary 3.4, for $r \gg 0$,

$$
T_{f}(r)=\max _{i} \log _{\pi}\left|P_{i}\right|_{r}+O(1)=D \log _{\pi} r+O(1),
$$

as was to be shown.

If $f$ is constant, then clearly $T_{f}$ is bounded. Thus (iii) implies (i). To show (iii), let $f$ be non-constant, and let $\left(f_{0}, \ldots, f_{n}\right)$ be analytic coordinate functions for $f$ without common factors. Since $f$ is not constant, at least one of these coordinate functions has a zero by Corollary 2.4. Therefore

$$
T_{f}(r) \geq N_{f_{0}}(0, r)+O(1) \geq \log _{\pi} r+O(1)
$$

for $r \gg 0$.

\section{Logarithmic Derivative Lemmas}

If $\gamma$ is a multi-index and $f$ is a meromorphic function of $m$ variables, then denote by $\partial^{\gamma} f$ the partial derivative

$$
\frac{\partial^{|\gamma|} f}{\partial z_{1}^{\gamma_{1}} \ldots \partial z_{m}^{\gamma_{m}}}
$$

The following important lemma says that the mean-proximity function is insignificant for logarithmic derivatives. 
Lemma 4.1. Let $f$ be a meromorphic function on $\mathbf{F}^{m}$, and let $\gamma$ be a multi-index with $|\gamma|>0$. Then,

$$
\left|\frac{\partial^{\gamma} g}{g}\right|_{r} \leq \frac{1}{r^{|\gamma|}}
$$

and in particular, for all $r \geq 1$,

$$
m_{\partial \gamma f / f}(\infty, r)=0 .
$$

Remark. Since for $r \geq 1, \log _{\pi}(1 / r) \leq 0$, the first statement is stronger than the second, and this is what gives Khoái-Tu $[\mathrm{K}-\mathrm{T}]$ the better error term they need for their applications.

Proof. Let

$$
g=\sum_{\eta} c_{\eta} z^{\eta}
$$

be an analytic function. Then

$$
\partial^{\gamma} g=\sum_{\eta \geq \gamma} c_{\eta}[\eta \cdots(\eta-\gamma+1)] z^{\eta-\gamma},
$$

where we have the understandings

$$
\begin{gathered}
\eta \geq \gamma \text { if } \eta_{i} \geq \gamma_{i} \text { for all } i, \quad \eta-\gamma=\left(\eta_{1}-\gamma_{1}, \ldots, \eta_{m}-\gamma_{m}\right), \\
\text { and }[\eta \cdots(\eta-\gamma+1)]=\prod_{i=1}^{m}\left(\eta_{i}\right)\left(\eta_{i}-1\right) \cdots\left(\eta_{i}-\gamma_{i}+1\right) .
\end{gathered}
$$

Because $[\eta \cdots(\eta-\gamma+1)]$ is an integer, and all integers have absolute value $\leq 1$,

$$
\begin{aligned}
\left|\partial^{\gamma} g\right|_{r} & =\sup _{\eta \geq \gamma}\left|c_{\eta}\right|_{v}|[\eta \cdots(\eta-\gamma+1)]|_{v} r^{|\eta|-|\gamma|} \\
& \leq \sup _{\eta \geq \gamma}\left|c_{\eta}\right|_{v} r^{|\eta|-|\gamma|} \\
& \leq \sup _{\eta}\left|c_{\eta}\right|_{v} \frac{r^{|\eta|}}{r^{|\gamma|}} \\
& =\frac{|g|_{r}}{r^{|\gamma|}}
\end{aligned}
$$

This implies that

$$
\left|\frac{\partial^{\gamma} g}{g}\right|_{r} \leq \frac{1}{r^{|\gamma|}}
$$

If $f$ is meromorphic, write $f=g / h$, where $g$ and $h$ are analytic. If $|\gamma|=1$, then

$$
\frac{\partial^{\gamma} f}{f}=\frac{\partial^{\gamma} g}{g}-\frac{\partial^{\gamma} h}{h}
$$

and so

$$
\left|\frac{\partial^{\gamma} f}{f}\right|_{r} \leq \max \left\{\left|\frac{\partial^{\gamma} g}{g}\right|_{r},\left|\frac{\partial^{\gamma} h}{h}\right|_{r}\right\} \leq \frac{1}{r}
$$

from above since $g$ and $h$ are analytic. If $k=|\gamma|>1$, then let $\gamma_{1}, \ldots, \gamma_{k-1}$ be multi-indices such that $\left|\gamma_{1}\right|=1$ and

$$
\gamma_{1}<\cdots<\gamma_{k-1}<\gamma \text {. }
$$


Then

$$
\frac{\partial^{\gamma} f}{f}=\frac{\partial^{\gamma_{1}} f}{f} \frac{\partial^{\gamma_{2}} f}{\partial^{\gamma_{1} f}} \cdots \frac{\partial^{\gamma} f}{\partial^{\gamma_{k-1} f}},
$$

where each term in the product is a logarithmic derivative of order 1, and so is covered by the previous case. The proof is completed by invoking the multiplicativity of ||$_{r}$.

A meromorphic map $f$ from $\mathbf{F}^{m}$ to $\mathbf{P}^{n}$ is called linearly non-degenerate if the image of $f$ is not contained in any hyperplane of $\mathbf{P}^{n}$. If $f=\left(f_{0}, \ldots, f_{n}\right)$ is an $n+1$-tuple of analytic functions and if $\gamma$ is a multi-index, then by $\partial^{\gamma} f$ we mean the $n+1$-tuple $\left(\partial^{\gamma} f_{0}, \ldots, \partial^{\gamma} f_{n}\right)$.

Lemma 4.2. Let $f$ be a linearly non-degenerate meromorphic map from $\mathbf{F}^{m}$ to $\mathbf{P}^{n}$ given by analytic coordinate functions $\left(f_{0}, \ldots, f_{n}\right)$. There exist multi-indices $\gamma_{1}, \ldots, \gamma_{n}$ such that $\left|\gamma_{i}\right| \leq i$ and such that the set of $\mathbf{F}^{n+1}$-valued functions

$$
f, \partial^{\gamma_{1}} f, \ldots, \partial^{\gamma_{n}} f
$$

is linearly independent over the field of meromorphic functions on $\mathbf{F}^{m}$.

Proof. The theory of "generalized Wronskians" as in $\S 4$ of Fujimoto [Fu] is easily seen to generalize to analytic functions on $\mathbf{F}^{m}$, so we leave the details of the proof as an exercise to the reader.

\section{Ramification and the Hyperplane Second Main Theorem}

Let $f$ be a linearly non-degenerate meromorphic map from $\mathbf{F}^{m}$ to $\mathbf{P}^{n}$, represented by analytic coordinate functions $\left(f_{0}, \ldots, f_{n}\right)$ that are without common factors. Let $\gamma_{1}, \ldots, \gamma_{n}$ be multi-indices such that $f, \partial^{\gamma_{1}} f, \ldots, \partial^{\gamma_{n}} f$ are linearly independent, as $\mathbf{F}^{n+1}$-valued functions. Lemma 4.2 guarantees we can always find such $\gamma_{i}$, and with $\left|\gamma_{i}\right| \leq i$. The linear non-degeneracy of $f$ implies that the "Wronskian"

$$
W=\operatorname{det}\left(\begin{array}{ccc}
f_{0} & \cdots & f_{n} \\
\partial^{\gamma_{1}} f_{0} & \cdots & \partial^{\gamma_{1}} f_{n} \\
& \vdots & \\
\partial^{\gamma_{n}} f_{0} & \cdots & \partial^{\gamma_{n}} f_{n}
\end{array}\right)
$$

is not identically zero. For a linearly non-degenerate meromorphic map, define the ramification term $N_{f, \operatorname{Ram}}(r)$ by

$$
N_{f, \operatorname{Ram}}(r)=N_{W}(0, r) .
$$

For different choices of the $\gamma_{i}$ one gets different ramification terms.

Theorem 5.1. Second Main Theorem. Let $H_{1}, \ldots, H_{q}$ be $q$ hyperplanes in general position in $\mathbf{P}^{n}$. Let $f$ be a linearly non-degenerate meromorphic map from $\mathbf{F}^{m}$ to $\mathbf{P}^{n}$, represented by analytic coordinate functions $\left(f_{0}, \ldots, f_{n}\right)$ without common factors. Let $\gamma_{1}, \ldots, \gamma_{n}$ be multi-indices such that the "Wronskian"

$$
W=W\left(f_{0}, \ldots, f_{n}\right)=\operatorname{det}\left(\begin{array}{ccc}
f_{0} & \cdots & f_{n} \\
\partial^{\gamma_{1}} f_{0} & \cdots & \partial^{\gamma_{1}} f_{n} \\
& \vdots & \\
\partial^{\gamma_{n}} f_{0} & \ldots & \partial^{\gamma_{n}} f_{n}
\end{array}\right)
$$


is not identically zero. Let

$$
B=\sum_{i=1}^{n}\left|\gamma_{i}\right|
$$

and let $r_{0}$ be a positive real number. Then, for all $r \geq r_{0}$, we have

$$
\sum_{j=1}^{q} m_{f}\left(H_{j}, r\right)-(n+1) T_{f}(r)+N_{f, \operatorname{Ram}}(r) \leq-B \log _{\pi} r+O(1),
$$

where the $O(1)$ term depends only on the hyperplanes, the map $f$, and $r_{0}$.

Remark. The $-(n+1)$ in front of $T_{f}(r)$ is significant because the degree of the canonical bundle on $\mathbf{P}^{n}$ is $-(n+1)$. Lemma 4.2 assures us that we can always find multi-indices $\gamma_{i}$ as in the theorem, and that moreover we can find these $\gamma_{i}$ with $1 \leq\left|\gamma_{i}\right| \leq i$, in which case $n \leq B \leq n(n+1) / 2$.

Remark. In our proof of this theorem, we essentially follow Lang's [La] presentation of Cartan's [Ca] proof, as in [Ch 1]. However, we also incorporate Lemma 4.2 to generalize the proof to several variables, and instead of using the Lemma on the Logarithmic Derivative, we use the stronger statement in Lemma 4.1. The importance of replacing the Logarithmic Derivative Lemma with this stronger estimate was recognized by Khoái-Tu $[\mathrm{K}-\mathrm{T}]$, and this is what results in the $-B \log _{\pi} r$ term on the right of the inequality. This extra term turns out to be crucial for the applications in $[\mathrm{K}-\mathrm{T}]$.

Proof. Since any collection of less than $n+2$ hyperplanes in general position can be extended to a set of $n+2$ hyperplanes in general position, and since for $r \geq r_{0}$, $m_{f}\left(H_{j}, r\right) \geq-O(1)$, it suffices to consider $q \geq n+2$. Let $x=\left(x_{0}, \ldots, x_{n}\right)$ be the projective coordinates on $\mathbf{P}^{n}$, and let $\left(f_{0}, \ldots, f_{n}\right)$ be analytic coordinate functions without common factors representing $f$. By abuse of notation, let

$$
H_{j}(x)=\sum_{k=0}^{n} a_{j k} x_{k}
$$

denote a linear form defining the hyperplane $H_{j}$.

First we claim that by general position of the hyperplanes, there exists a constant $C$, depending only on the hyperplanes $H_{j}$ and our choice of projective coordinates, such that for each $r$, there are at most $n$ hyperplanes $H_{j}$ that do not satisfy the following inequality:

$$
\max _{k} \log _{\pi}\left|f_{k}\right|_{r}-C \leq \log _{\pi}\left|H_{j} \circ f\right|_{r} \leq \max _{k} \log _{\pi}\left|f_{k}\right|_{r}+C .
$$

Indeed, for the right hand inequality, we need only take $C$ to be larger than $\log _{\pi}\left|a_{j k}\right|_{v}$ for all $j$ and $k$. For the left hand inequality, let $J \subset\{1, \ldots, q\}$ have cardinality $n+1$, and choose $K$ so that

$$
\left|f_{K}\right|_{r}=\max _{k}\left|f_{k}\right|_{r} .
$$

By general position of the hyperplanes, we can write

$$
f_{k}=\sum_{j \in J} b_{k j}^{(J)} H_{j} \circ f
$$


and so

$$
\max _{k} \log _{\pi}\left|f_{k}\right|_{r}=\log _{\pi}\left|f_{K}\right|_{r} \leq \log _{\pi}\left|H_{j} \circ f\right|_{r}+C
$$

for at least one index $j$ in $J$ provided that $C$ is larger than $\log _{\pi}\left|b_{K j}^{(J)}\right|_{v}$ for all $j$. As there are only finitely many possible index sets $J$, we can choose $C$ so large that it works for all the $b_{k j}^{(J)}$.

Now, let $s=q-(n+1)$, and let $M=\left\{m_{1}, \ldots, m_{s}\right\}$ be a subset of $\{1, \ldots, q\}$ with cardinality $s$. For such a subset $M$, let

$$
H_{M}(x)=H_{m_{1}}(x) \cdots H_{m_{s}}(x) .
$$

Corollary 3.4 and inequality $(*)$ give us

$$
s T_{f}(r)=s \max _{j} \log _{\pi}\left|f_{j}\right|_{r}+O(1)=\max _{M} \log _{\pi}\left|H_{M} \circ f\right|_{r}+O(1) .
$$

Let $h_{j}=H_{j} \circ f$. Let $I=\left\{i_{0}, \ldots, i_{n}\right\} \subset\{1, \ldots, q\}$ denote a set of indices of cardinality $n+1$. Given $n+1$ analytic functions $\left(g_{0}, \ldots, g_{n}\right)$ on $\mathbf{F}^{m}$, we will use $W\left(g_{0}, \ldots, g_{n}\right)$ to denote the "Wronskian" of those functions with respect to the $\gamma_{i}$ as in the statement of the theorem. Note that if $d_{I}$ is the determinant of the linear transformation taking the standard projective coordinates on $\mathbf{P}^{n}$ to the hyperplanes $H_{i_{0}}, \ldots, H_{i_{n}}$, then

$$
W\left(h_{i_{0}}, \ldots, h_{i_{n}}\right)=d_{I} W\left(f_{0}, \ldots, f_{n}\right)
$$

Decompose $\{1, \ldots, q\}$ into a disjoint union $I \cup M$, where $I$ has cardinality $n+1$ and $M$ has cardinality $s$. Let

$$
L_{I}=\frac{W\left(h_{i_{0}}, \ldots, h_{i_{n}}\right)}{h_{i_{0}} \cdots h_{i_{n}}} \quad \text { and } \quad G=\frac{h_{1} \ldots h_{q}}{W\left(f_{0}, \ldots, f_{n}\right)} .
$$

Then,

$$
H_{M} \circ f=h_{m_{1}} \cdots h_{m_{s}}=G d_{I} L_{I} .
$$

The term $L_{I}$ is a sum of products of logarithmic derivatives. Within each product, there are exactly $n$ logarithmic derivatives, one of order $\left|\gamma_{k}\right|$ for each $k$ between 1 and $n$. Therefore by Lemma 4.1,

$$
\max _{I} \log _{\pi}\left|L_{I}\right|_{r} \leq-B \log _{\pi} r
$$

As for the $G$ term, the Poisson-Jensen-Green formula (Theorem 3.1) and the definition of $G$ give us

$$
\begin{aligned}
\log _{\pi}|G|_{r} & =N_{G}(0, r)-N_{G}(\infty, r)+O(1) \\
& =\sum_{j=1}^{q}\left(N_{h_{j}}(0, r)-N_{h_{j}}(\infty, r)\right)-N_{W}(0, r)+N_{W}(\infty, r)+O(1) \\
& =\sum_{j=1}^{q} N_{h_{j}}(0, r)-N_{W}(0, r)+O(1),
\end{aligned}
$$


where the last line follows since $h_{j}$ and $W$ are analytic. Hence

$$
\begin{aligned}
s T_{f}(r) & =\max _{M} \log _{\pi}\left|H_{M} \circ f\right|_{r}+O(1) \\
& =\max _{I} \log _{\pi}\left|G L_{I}\right|_{r}+O(1) \\
& \leq \sum_{j=1}^{q} N_{f}\left(H_{j}, r\right)-N_{W}(0, r)-B \log _{\pi} r+O(1) .
\end{aligned}
$$

The theorem follows by recalling that $N_{f, \operatorname{Ram}}(r)=N_{W}(0, r)$ and that

$$
\begin{aligned}
s T_{f}(r)-\sum_{j=1}^{q} N_{f}\left(H_{j}, r\right) & =\sum_{j=1}^{q} m_{f}\left(H_{j}, r\right)-(q-s) T_{f}(r)+O(1) \\
& =\sum_{j=1}^{q} m_{f}\left(H_{j}, r\right)-(n+1) T_{f}(r)+O(1) .
\end{aligned}
$$
be

If we define the defect $\delta_{f}(H)$ of a hyperplane $H$ for a meromorphic map $f$ to

$$
\delta_{f}(H)=\liminf _{r \rightarrow \infty}\left\{1-\frac{N_{f}(H, r)}{T_{f}(r)}\right\},
$$

then we immediately get the following corollary:

Corollary 5.2. Defect Relation. Let $f$ be a linearly non-degenerate meromorphic map from $\mathbf{F}^{m}$ to $\mathbf{P}^{n}$. Let $H_{1}, \ldots, H_{q}$ be $q$ hyperplanes in general position. Then

$$
\sum_{j=1}^{q} \delta_{f}\left(H_{j}\right) \leq n+1 .
$$

\section{Defect Relations and Inverse Problems}

In this section we examine defects more closely. We show that the defect relation (Corollary 5.2) from the Second Main Theorem (Theorem 5.1) is not sharp, by proving that there can be at most $n$ hyperplanes in general position that have nonzero defects. We then construct examples to show that this is the only restriction on defects. Finally, we give a few easy examples to show that the Second Main Theorem with the ramification term is sharp.

Theorem 6.1. Let $f$ be a linearly non-degenerate meromorphic map from $\mathbf{F}^{m}$ to $\mathbf{P}^{n}$. Let $H_{1}, \ldots, H_{q}$ be $q$ hyperplanes in general position such that

$$
\delta_{f}\left(H_{j}\right)>0 \text { for all } j .
$$

Then $q \leq n$.

Since $0 \leq \delta_{f}(H) \leq 1$ for any hyperplane $H$, this immediately implies

Corollary 6.2. If $H_{1}, \ldots, H_{q}$ are $q$ hyperplanes in general position, then

$$
\sum_{j=1}^{q} \delta_{f}\left(H_{j}\right) \leq n
$$


Proof of Theorem 6.1. It suffices to show that $q$ cannot be $n+1$. Since the hyperplanes are in general position, we can, by making a linear change of coordinates on $\mathbf{P}^{n}$, assume that the hyperplanes are the coordinate hyperplanes. Let $f_{0}, \ldots, f_{n}$ be analytic coordinate functions representing $f$. By assumption,

$$
\liminf _{r \rightarrow \infty}\left\{\frac{m_{f}\left(H_{j}, r\right)}{T_{f}(r)}\right\}=\liminf _{r \rightarrow \infty}\left\{1-\frac{N_{f}\left(H_{j}, r\right)}{T_{f}(r)}\right\}=\delta_{f}\left(H_{j}\right)>0 .
$$

This implies that for all $r$ sufficiently large

$$
m_{f}\left(H_{j}, r\right)>0 \text { for all } j .
$$

By the definition of $m_{f}\left(H_{j}, r\right)$,

$$
\max _{i} \log _{\pi}\left|f_{i} / f_{j}\right|_{r}>0 \text { for all } j .
$$

Clearly this is absurd.

We now proceed to show that Theorem 6.1 is the only restriction on defects. We begin with a lemma.

Lemma 6.3. Let $0<\varepsilon \leq 1$. Let $\rho$ be a positive real number. Let $S$ be the largest integer $k$ such that $[k / \varepsilon] \leq \rho$, where for any real number $x,[x]$ denotes the greatest integer $\leq x$. Then

$$
S \rho-\sum_{k=1}^{S}[k / \varepsilon]=\varepsilon \frac{\rho^{2}}{2}+O(\rho) .
$$

Proof. By definition

$$
\frac{k}{\varepsilon}-1<\left[\frac{k}{\varepsilon}\right] \leq \frac{k}{\varepsilon}
$$

so clearly

$$
\varepsilon \rho-1<S<\varepsilon(\rho+1) .
$$

Therefore,

$$
\varepsilon \rho^{2}-\rho<S \rho<\varepsilon\left(\rho^{2}+\rho\right) .
$$

Now,

$$
\sum_{k=1}^{S}\left[\frac{k}{\varepsilon}\right] \leq \frac{1}{\varepsilon} \sum_{k=1}^{S} k=\frac{1}{\varepsilon} \frac{S(S+1)}{2} \leq \varepsilon \frac{\rho^{2}}{2}+O(\rho),
$$

where the last inequality follows from $(*)$. Similarly,

$$
\sum_{k=1}^{S}\left[\frac{k}{\varepsilon}\right]>\frac{1}{\varepsilon} \sum_{k=1}^{S} k-S=\frac{1}{\varepsilon} \frac{S(S+1)}{2}-S>\varepsilon \frac{\rho^{2}}{2}+O(\rho) .
$$

Hence,

$$
\sum_{k=1}^{S}\left[\frac{k}{\varepsilon}\right]=\varepsilon \frac{\rho^{2}}{2}+O(\rho) .
$$

The lemma follows by combining this with $(* *)$. 
Theorem 6.4. Let $m$ and $n$ be positive integers. Let $q$ be a positive integer less than or equal to $n$. Let $H_{1}, \ldots, H_{q}$ be q linearly independent hyperplanes in $\mathbf{P}^{n}$. Let $0<\delta_{j} \leq 1$ for $j=1, \ldots, q$ be real numbers. There exists a linearly non-degenerate meromorphic map $f$ from $\mathbf{F}^{m}$ to $\mathbf{P}^{n}$ such that $\delta_{f}\left(H_{j}\right)=\delta_{j}$ for $j=1, \ldots, q$, and such that $\delta_{f}(H)=0$ for any hyperplane $H$ that is in general position with (i.e. linearly independent with) $H_{1}, \ldots, H_{q}$. Moreover, if $m \geq n$, we can choose $f$ so its Jacobian has maximal rank.

Proof. First note that it suffices to prove the theorem when $H_{1}, \ldots, H_{q}$ are the coordinate hyperplanes given by $x_{i}=0, i=1, \ldots, q$, for a set of projective coordinates $\left(x_{1}, \ldots, x_{n+1}\right)$. We can also assume that $\delta_{1} \geq \cdots \geq \delta_{q}$. Let $p$ be the integer (possibly zero) such that $\delta_{j}=1$ if $j \leq p$, and $\delta_{j}<1$ if $j>p$. Note that in the sequel if $p=0$, then all statements holding for $j=1, \ldots, p$ will be taken to be vacuous.

We begin by defining $n+1$ analytic functions of one variable, $g_{j}(z)$. Let $b$ be an element of $\mathbf{F}$ such that $|b|_{v}>1$. Let $a_{1}, \ldots, a_{n+1}$ be $n+1$ distinct elements of $\mathbf{F}$ such that $\left|a_{j}\right|_{v}=1$ for all $j$. For $j=1, \ldots, p$, let

$$
g_{j}(z)=\left(z-a_{j}\right)^{j}
$$

For $j=p+1, \ldots, n+1$, let

$$
g_{j}(z)=\prod_{i=1}^{\infty}\left(1-a_{j} \frac{z}{b^{\left[i /\left(1-\delta_{j}\right)\right]}}\right),
$$

where again $[x]$ denotes the greatest integer less than or equal to $x$ for any real number $x$, and where $\delta_{j}=0$ for $j>q$. Because $|b|_{v}>1$ and $\left|a_{j}\right|_{v}=1$, Lemma 2.1 implies that each of the $g_{j}$ is an analytic function on $\mathbf{F}$.

For $j=1, \ldots, \min \{m, n+1\}$, let

$$
f_{j}\left(z_{1}, \ldots, z_{m}\right)=z_{j}^{j-1} g_{j}\left(z_{j}^{n+1}\right)
$$

and if $m<n+1$, then for $j=m+1, \ldots, n+1$, let

$$
f_{j}\left(z_{1}, \ldots, z_{m}\right)=z_{m}^{j-1} g_{j}\left(z_{m}^{n+1}\right) \text {. }
$$

Note that we have only used one variable in the definition of each $f_{j}$. Since we chose the $a_{j}$ to be distinct, the $g_{j}$ do not have any common zeros by construction. The $f_{j}$ differ from the $g_{j}$ only by powers of $z_{j}$ or $z_{m}$, and since $f_{1}(0) \neq 0$, the $f_{i}$ are also analytic without common factors (since considered as functions of one variable they have no common zeros). Note that we have constructed the $f_{j}$ so that the exponents in all the non-zero terms of the power series expansion of $f_{j}$ are congruent to $j-1$ modulo $n+1$, and so in particular the set of exponents in the power series expansion for $f_{j}$ is disjoint from the set of exponents in the power series expansion for $f_{k}$ whenever $j \neq k$.

Let $f$ be the map from $\mathbf{F}^{m}$ to $\mathbf{P}^{n}$ given by the coordinate functions $f_{j}$. Note that if $m \geq n$, then we have used a different variable for at least $n$ of the coordinate functions; hence $f$ has maximal rank Jacobian, and so in particular, $f$ is linearly non-degenerate. In any case, as the sets of exponents in the series expansions for each of $f_{j}$ are pairwise disjoint, $f$ is linearly non-degenerate even when $m<n$.

We will now compute $\log _{\pi}\left|g_{j}\right|_{r}$ for each $j$. Fix $r>1$. For $j=1, \ldots, p$,

$$
\log _{\pi}\left|g_{j}\right|_{r}=j \log _{\pi} r
$$


For $j=p+1, \ldots, n+1$ (where again we take $\delta_{j}=0$ if $j>q$ ),

$$
\log _{\pi}\left|g_{j}\right|_{r}=S_{j} \log _{\pi} r-\sum_{i=1}^{S_{j}}\left[\frac{i}{1-\delta_{j}}\right] \log _{\pi}|b|_{v}
$$

where $S_{j}$ is the largest integer $i$ such that

$$
\left[\frac{i}{1-\delta_{j}}\right] \log _{\pi}|b|_{v} \leq \log _{\pi} r
$$

Setting

$$
\varepsilon=1-\delta_{j} \quad \text { and } \quad \rho=\frac{\log _{\pi} r}{\log _{\pi}|b|_{v}}
$$

in Lemma 6.3 gives us

$$
\log _{\pi}\left|g_{j}\right|_{r}=\left(1-\delta_{j}\right) \frac{\left(\log _{\pi} r\right)^{2}}{2 \log _{\pi}|b|_{v}}+O\left(\log _{\pi} r\right), \quad j=p+1, \ldots, n+1 .
$$

Also, since $\delta_{n+1}=0$,

$$
\max _{1 \leq j \leq n+1}\left|g_{j}\right|_{r}=\frac{\left(\log _{\pi} r\right)^{2}}{2 \log _{\pi}|b|_{v}}+O\left(\log _{\pi} r\right) .
$$

Now we will check that $f$ has the specified deficiencies. Since the $f_{j}$ are without common factors, Corollary 3.4 and the above estimates on $\left|g_{j}\right|_{r}$ then tell us that

$$
\begin{aligned}
T_{f}(r) & =\max _{j} \log _{\pi}\left|f_{j}\right|_{r}+O(1) \\
& =\max _{j} \log _{\pi}\left|g_{j}\right|_{r^{n+1}}+O\left(\log _{\pi} r\right) \\
& =\frac{(n+1)^{2}\left(\log _{\pi} r\right)^{2}}{2 \log _{\pi}|b|_{v}}+O\left(\log _{\pi} r\right) .
\end{aligned}
$$

We note in passing that $f$ has growth order 0 . Now, directly from the definition, for $j=1, \ldots, p$,

$$
\begin{aligned}
m_{f}\left(H_{j}, r\right) & =\max _{i} \log _{\pi}\left|f_{i}\right|_{r}-\log _{\pi}\left|f_{j}\right|_{r} \\
& =\max _{i} \log _{\pi}\left|g_{i}\right|_{r^{n+1}}+O\left(\log _{\pi} r\right) \\
& =\frac{(n+1)^{2}\left(\log _{\pi} r\right)^{2}}{2 \log _{\pi}|b|_{v}}+O\left(\log _{\pi} r\right),
\end{aligned}
$$

and, for $j=p+1, \ldots, q$,

$$
\begin{aligned}
m_{f}\left(H_{j}, r\right) & =\max _{i} \log _{\pi}\left|f_{i}\right|_{r}-\log _{\pi}\left|f_{j}\right|_{r} \\
& =\max _{i} \log _{\pi}\left|g_{i}\right|_{r^{n+1}}-\log _{\pi}\left|g_{j}\right|_{r^{n+1}}+O\left(\log _{\pi} r\right) \\
& =\delta_{j} \frac{(n+1)^{2}\left(\log _{\pi} r\right)^{2}}{2 \log _{\pi}|b|_{v}}+O\left(\log _{\pi} r\right) .
\end{aligned}
$$

Therefore, for $j=1, \ldots, q$,

$$
\delta_{f}\left(H_{j}\right)=\liminf _{r \rightarrow \infty} \frac{m_{f}\left(H_{j}, r\right)}{T_{f}(r)}=\delta_{j} .
$$

To complete the proof, we need to check that if $H$ is any hyperplane in general position with $H_{1}, \ldots, H_{q}$, then $\delta_{f}(H)=0$. Since $H$ is assumed linearly independent with $H_{1}, \ldots, H_{q}$, the linear form $H(x)=\sum c_{j} x_{j}$ defining $H$ is such that $c_{j} \neq 0$ 
for at least one $j>q$, and so without loss of generality we assume that $c_{n+1} \neq 0$. Because the sets of exponents in the power series expansions of the $f_{j}$ are pairwise disjoint, we see that

$$
\left|\sum_{j=1}^{n+1} c_{j} f_{j}\right|_{r}=\max _{j}\left|c_{j}\right|_{v}\left|f_{j}\right|_{r} .
$$

Thus, for all $r$ sufficiently large,

$$
\log _{\pi}|H \circ f|_{r}=\max _{j} \log _{\pi}\left|c_{j} f_{j}\right|_{r}=\log _{\pi}\left|f_{n+1}\right|_{r}+O\left(\log _{\pi} r\right),
$$

and this implies that for all $r$ sufficiently large,

$$
m_{f}(H, r)=\max _{j} \log _{\pi}\left|f_{j}\right|_{r}-\log _{\pi}|H \circ f|_{r}+O(1)=O\left(\log _{\pi} r\right) .
$$

Thus $\delta_{f}(H)=0$, and the proof is complete.

As previously mentioned, the fact that the defect relation in Corollary 5.2 is not sharp does not mean that the Second Main Theorem (Theorem 5.1) itself is not sharp, because Theorem 5.1 also contains a ramification term on the left-hand side. We now give an easy example to show that with the ramification term, Theorem 5.1 is sharp in the following sense. If $f$ is a linearly non-degenerate meromorphic map from $\mathbf{F}^{m}$ to $\mathbf{P}^{n}$, then define the total ramification defect $\Theta_{f}$ to be

$$
\Theta_{f}=\liminf _{r \rightarrow \infty} \frac{N_{f, \operatorname{Ram}}(r)}{T_{f}(r)} .
$$

Theorem 6.5. Let $m$ and $n$ be positive integers. There exist a linearly nondegenerate meromorphic map $f=\left(f_{0}, \ldots, f_{n}\right): \mathbf{F}^{m} \rightarrow \mathbf{P}^{n}$ and a collection of hyperplanes $H_{1}, \ldots, H_{q}$ in general position such that

$$
\sum_{j=1}^{q} \delta_{f}\left(H_{j}\right)+\Theta_{f}=n+1 .
$$

Moreover, if $m \geq n$, we can choose $f$ so that we may take

$$
N_{f, \operatorname{Ram}}(r)=N_{J}(0, r),
$$

where $J$ is the following Jacobian:

$$
J=\operatorname{det}\left(\begin{array}{cccc}
f_{0} & f_{1} & \ldots & f_{n} \\
\frac{\partial f_{0}}{\partial z_{1}} & \frac{\partial f_{1}}{\partial z_{1}} & \ldots & \frac{\partial f_{n}}{\partial z_{1}} \\
\frac{\partial f_{0}}{\partial z_{n}} & \frac{\partial f_{1}}{\partial z_{n}} & \ldots & \frac{\partial f_{n}}{\partial z_{n}}
\end{array}\right) .
$$

Remark. To require that a map have Jacobian not identically zero is a much stronger non-degeneracy condition than linear non-degeneracy. The point of the second statement in the above theorem is to show that the Second Main Theorem is also sharp if one adds the stronger non-degeneracy condition as a hypothesis.

Before giving a proof of Theorem 6.5, we first need the following lemma. 
Lemma 6.6. Let $n$ be a positive integer. There exists a transcendental analytic function $f$ on $\mathbf{F}$ such that

$$
T_{f^{(n)}}(r)=T_{f}(r)+O\left(\log _{\pi} r\right) .
$$

Proof. If the characteristic of $\widetilde{\mathbf{F}}$, the residue class field of $\mathbf{F}$, is zero, then the lemma is trivial. For in that case, let

$$
f=\sum a_{j} z^{j}
$$

be any transcendental analytic function. For $r$ sufficiently large,

$$
\left|f^{(n)}\right|_{r}=\frac{|f|_{r}}{r^{n}} .
$$

Therefore, for $r$ sufficiently large,

$$
T_{f^{(n)}}(r)=m_{f^{(n)}}(\infty, r)+O(1)=m_{f}(\infty, r)+O\left(\log _{\pi} r\right)=T_{f}(r)+O\left(\log _{\pi} r\right),
$$

and we are done.

Now assume that the characteristic of $\widetilde{F}$ is different from zero. For each positive integer $k$, let

$$
I_{k}=\left\{i \in \mathbf{Z}:|i|_{v} \leq \pi^{-k}\right\} .
$$

Clearly $I_{k}$ is an ideal, and it is not the zero ideal because of our assumption on the characteristic of the residue class field. It is easy to see that the cardinality of $\mathbf{Z} / I_{k}$ tends to infinity as $k$ tends to infinity. Let $K$ be an integer sufficiently large so that the cardinality of $\mathbf{Z} / I_{K}$ is larger than $n$. Let $i$ be the positive generator of $I_{K}$. Let

$$
f=\sum_{j} a_{j} z^{i j+n}
$$

where the $a_{j}$ are non-zero coefficients chosen small enough to ensure that $f$ is analytic on all of $\mathbf{F}$. Trivially,

$$
f^{(n)}=\sum_{j} a_{j}(i j+n) \cdots(i j+1) z^{i j} .
$$

Because we chose $K$ large enough, none of $(i j+1), \ldots,(i j+n)$ are in $I_{K}$. Therefore, each of these numbers has norm greater than $\pi^{-K}$, and hence

$$
|(i j+1) \cdots(i j+n)|_{v}>\pi^{-n K} .
$$

Thus for $r$ sufficiently large,

$$
\left|f^{(n)}\right|_{r} \geq \pi^{-n K} \frac{|f|_{r}}{r^{n}} .
$$

On the other hand, since $|j|_{v} \leq 1$ for all integers $j$,

$$
\left|f^{(n)}\right|_{r} \leq \frac{|f|_{r}}{r^{n}}
$$

for $r$ sufficiently large. Therefore,

$$
T_{f^{(n)}}(r)=T_{f}(r)+O\left(\log _{\pi} r\right),
$$

as in the case when char $\widetilde{\mathbf{F}}=0$. 
Proof of Theorem 6.5. First we give an example for arbitrary $m$ and $n$ using a Wronskian for the ramification term. Let $g(z)$ be a transcendental analytic function in one variable such that

$$
T_{g^{(n)}}(r)=T_{g}(r)+O\left(\log _{\pi} r\right) .
$$

Lemma 6.6 ensures the existence of such a $g$. Consider the meromorphic map from $\mathbf{F}^{m}$ to $\mathbf{P}^{n}$ given by

$$
\left(1, z_{1}, z_{1}^{2}, \ldots, z_{1}^{n-1}, g\left(z_{1}\right)\right)
$$

where $z_{1}$ is the first variable on $\mathbf{F}^{m}$. If we write out the Wronskian, we get

$$
W=\operatorname{det}\left(\begin{array}{ccccc}
1 & z_{1} & z_{1}^{2} & \ldots & g\left(z_{1}\right) \\
0 & 1 & 2 z_{1} & \ldots & g^{\prime}\left(z_{1}\right) \\
0 & 0 & 2 & \ldots & g^{\prime \prime}\left(z_{1}\right) \\
& & & \vdots & \\
0 & 0 & 0 & \ldots & g^{(n)}\left(z_{1}\right)
\end{array}\right) .
$$

Clearly $W$ is a constant multiple of $g^{(n)}$. Because $W$ is analytic, for $r$ sufficiently large, $m_{W}(0, r)=0$. Therefore for $r$ sufficiently large,

$$
\begin{aligned}
N_{W}(0, r) & =T_{W}(r)-m_{W}(0, r)+O(1) \\
& =T_{g^{(n)}}(r)+O(1) \\
& =T_{g}(r)+o\left(T_{g}(r)\right)=T_{f}(r)+o\left(T_{f}(r)\right) .
\end{aligned}
$$

Thus in this case $\Theta_{f}=1$. Also, the deficiencies of the first $n$ coordinate hyperplanes are each clearly 1 . Summing these defects together gives $n+1$, which is what we wanted to show.

If $m \geq n$ and we want an example with a Jacobian type ramification term rather than a Wronskian type ramification term, we need only do the following. This time let $g$ be a transcendental analytic function of one variable such that

$$
T_{g^{\prime}}(r)=T_{g}(r)+O\left(\log _{\pi} r\right) .
$$

Let $f$ be the meromorphic map from $\mathbf{F}^{m}$ to $\mathbf{P}^{n}$ given by

$$
\left(1, g\left(z_{1}\right), \ldots, g\left(z_{n}\right)\right)
$$

where $z_{1}, \ldots, z_{n}$ are the first $n$ coordinates on $\mathbf{F}^{m}$. Then

$$
J=g^{\prime}\left(z_{1}\right) \cdots g^{\prime}\left(z_{n}\right),
$$

and because $J$ is analytic, we again have $m_{J}(0, r)=0$ for $r$ sufficiently large. Thus,

$$
N_{J}(0, r)=T_{J}(0, r)-m_{J}(0, r)+O(1)=n T_{g}(r)+o\left(T_{g}(r)\right) .
$$

Thus $\Theta_{f}=n$. In addition, the first coordinate hyperplane clearly has defect 1 , so the sum of the defects, including the total ramification defect, is again $n+1$.

We conclude this paper with the following open question: 
Question 6.7. Let $m$ and $n$ be positive integers. Let $q$ be an integer such that $0 \leq q \leq n$. Let $H_{1}, \ldots, H_{q}$ be $q$ linearly independent hyperplanes (this set is empty if $q=0)$. Let $\delta_{1}, \ldots, \delta_{q}$ be $q$ real numbers such that $0<\delta_{j} \leq 1$. Let $0 \leq \Theta \leq n+1$ be a real number. Assume further that

$$
\sum_{j=1}^{q} \delta_{j}+\Theta \leq n+1 .
$$

Does there exist a linearly non-degenerate meromorphic map from $\mathbf{F}^{m}$ to $\mathbf{P}^{n}$ such that $\delta_{f}\left(H_{j}\right)=\delta_{j}$ for $j=1, \ldots, q$, such that $\Theta_{f}=\Theta$, and such that if $H$ is any hyperplane linearly independent with $H_{1}, \ldots, H_{q}$ then $\delta_{f}(H)=0$ ? If not, what additional restrictions are there on the $\delta_{f}(H)$ and $\Theta_{f}$ ?

\section{Appendix}

The argument presented in this appendix is due to W. Lütkebohmert [Lü].

Proposition 7.1. Greatest common divisors exist in the ring of analytic functions on $\mathbf{F}^{m}$.

Proof. Because $\mathbf{B}^{m}(r)$ is a unique factorization domain provided there exists an $a$ in $\mathbf{F}$ with $|a|_{v}=r$ (see [BGR] Theorem 5.2.6/1), then by standard arguments (see any book on several complex variables that discusses the Second Cousin Problem and the Poincaré Problem), we need only consider the following. Let $r_{i}$ be an increasing sequence of radii such that $r_{i} \rightarrow \infty$ and such that there exist $a_{i}$ in $\mathbf{F}$ with $\left|a_{i}\right|_{v}=r_{i}$. Let $B_{i}=\mathbf{B}^{m}\left(r_{i}\right)$, and let $f_{i}$ be analytic functions on $B_{i}$ such that for every $i$ and $j$ there exists an invertible analytic function $u_{i, j}$ on $\mathbf{B}^{m}\left(\min \left\{r_{i}, r_{j}\right\}\right)$ such that

$$
f_{i}=u_{i, j} f_{j} .
$$

We need to show there exists an analytic function $G$ on all of $\mathbf{F}^{m}$, such that on each $B_{i}$ there exist invertible analytic functions $v_{i}$ such that

$$
G=v_{i} f_{i} .
$$

If any of the $f_{i}$ are identically zero, then they all are, and the proposition is trivial in that case. So, let $z_{0}$ be a point in $B_{1}$ such that $f_{1}\left(z_{0}\right) \neq 0$, and therefore $f_{i}\left(z_{0}\right) \neq 0$ for all $i$. To construct $G$, we may assume, without loss of generality, that $f_{i}\left(z_{0}\right)=1$ for all $i$. For if not, then we just need to adjust the $v_{i}$ at the end by constant factors. The key point is that since $u_{i, i+1}$ is analytic and invertible on $B_{i}$, its power series expansion about $z_{0}$ is of the form

$$
u_{i, i+1}=1+\sum_{\gamma,|\gamma| \geq 1} a_{\gamma}\left(z-z_{0}\right)^{\gamma}, \quad \text { with }\left|a_{\gamma}\right|_{v} r_{i}^{|\gamma|}<1 \text { for all } \gamma .
$$

Hence, for $j>i$,

$$
\left|u_{j, j+1}-1\right|_{r_{i}}<\frac{r_{i}}{r_{j}}
$$

Keeping $i$ fixed and letting $j \rightarrow \infty$, we note that $r_{i} / r_{j} \rightarrow 0$, and so we can use infinite products (Proposition 2.1) to define

$$
v_{i}=\prod_{k=i}^{\infty} u_{k, k+1} .
$$


For $j>i$, note that

$$
\begin{aligned}
f_{j} v_{i} & =f_{j} \prod_{k=i}^{\infty} u_{k, k+1} \\
& =f_{j}\left(\prod_{k=i}^{j-1} u_{k, k+1}\right)\left(\prod_{k=j}^{\infty} u_{k, k+1}\right) \\
& =f_{i} v_{j}
\end{aligned}
$$

Therefore, $f_{i} v_{i}^{-1}=f_{j} v_{j}^{-1}$ on $B_{i}$ for all $j \geq i$. But, this means precisely that the functions $f_{i} v_{i}^{-1}$ converge to an analytic function $G$ on all of $\mathbf{F}^{m}$, and this $G$ is precisely the function we were looking for.

\section{REFERENCES}

[A-S] W. W. Adams and E. G. Straus, Non-Archimedean Analytic Functions Taking the Same Values at the Same Points, Illinois J. Math. 15 (1971), 418-424. MR 43:3504

[Ah] L. Ahlfors, The theory of meromorphic curves, Acta Soc. Sci. Finn., N.S., A, III (1941), 1-31. MR 2:357b

[Am] Y. Amice, Les nombres p-adiques, Presses Universitaires de France, 1975. MR 56:5510

[BGR] S. Bosch, U. Güntzer and R. Remmert, Non-Archimedean Analysis, Springer-Verlag, 1984. MR 86b:32031

[Bo 1] A. Boutabaa, Sur la théorie de Nevanlinna p-adique, Thése de Doctorat, Université Paris 7, 1991.

[Bo 2] A. Boutabaa, Theorie de Nevanlinna p-adique, Manuscripta Math. 67 (1990), 251-269. MR 91m:30039

[Bo 3] A. Boutabaa, Sur les courbes holomorphes p-adiques, Ann. Fac. Sci. Toulouse Math. (6) 5 (1996), 29-52. CMP 97:02

[C-G] J. Carlson and P. Griffiths, A defect relation for equidimensional holomorphic mappings between algebraic varieties, Ann. Math. 95 (1972), 557-584. MR 47:497

[Ca] H. Cartan, Sur les zéros des combinaisons linéaires de p fonctions holomorphes données, Mathematica 7 (1933), 5-31.

[Ch 1] W. Cherry, Hyperbolic p-Adic Analytic Spaces, Ph.D. Thesis, Yale University, 1993.

[Ch 2] W. Cherry, Non-Archimedean analytic curves in Abelian varieties, Math. Ann. 300 (1994), 393-404. MR 96i:14021

[Co 1] C. Corrales-Rodrigáñez, Nevanlinna Theory in the p-Adic Plane, Ph.D. Thesis, University of Michigan, 1986

[Co 2] C. Corrales-Rodrigáñez, Nevanlinna Theory on the p-Adic Plane, Annales Polonici Mathematici LVII (1992), 135-147. MR 93h:30067

[Dr] D. Drasin, The inverse problem of the Nevanlinna theory, Acta Math. 138 (1976), 83-151. MR 58:28502

[Fu] H. Fujimoto, Non-integrated defect relation for meromorphic maps of complete Kähler manifolds into $P^{N_{1}}(\mathbf{C}) \times \cdots \times P^{N_{k}}(\mathbf{C})$, Japan. J. Math. 11 (1985), 233-264. MR 88m:32049

[G-K] P. Griffiths and J. King, Nevanlinna theory and holomorphic mappings between algebraic varieties, Acta Math. 130 (1973), 145-220. MR 55:721

[Kh 1] Hà Huy Khoái, On p-Adic Meromorphic Functions, Duke Math. J. 50 (1983), 695-711. MR 85d:11092

[Kh 2] Hà Huy Khoái, Heights for p-Adic Meromorphic Functions and Value Distribution Theory, Max-Planck-Institut Für Mathematik. 89-67 (1989). Cf. MR 96j:32044

[Kh 3] Hà Huy Khoái, Heights for p-Adic Holomorphic Functions of Several Variables, MaxPlanck-Institut Für Mathematik. 89-83 (1989). Cf. MR 92f:32059

[Kn] H. Kneser, Zur Theorie der gebrochenen Funktionen mehrerer Veränderlicher, Jber. Deutsch. Math. Verein. 48 (1938/39), 1-28. 
[K-Q] Hà Huy Khoái and My Vinh Quang, On p-adic Nevanlinna Theory, in Lecture Notes in Mathematics 1351, Springer-Verlag 1988, pp. 146-158. MR 90e:11153

[K-T] Hà Huy Khoái and Mai Van Tu, p-Adic Nevanlinna-Cartan Theorem, Internat. J. Math. 6 (1995), 719-731. MR 96k:32053

[La] S. Lang, Introduction to Complex Hyperbolic Spaces, Springer-Verlag, 1987. MR 88f:32065

[L-C] S. Lang and W. Cherry, Topics in Nevanlinna Theory, Springer Lecture Notes in Mathematics 1433, Springer-Verlag, 1990. MR 91k:32025

[Lü] W. Lütkebohmert, Letter to R. Remmert, 1995.

[Ne 1] R. Nevanlinna, Le théorème de Picard-Borel et la théorie des fonctions méromorphes, Paris, 1929.

[Ne 2] R. Nevanlinna, Analytic Functions, Springer-Verlag, 1970. MR 43:5003

[Os 1] C.F. Osgood, A number theoretic-differential equations approach to generalizing Nevanlinna theory, Indian J. of Math. 23 (1981), 1-15. MR 85b:30043

[Os 2] C.F. Osgood, Sometimes effective Thue-Siegel-Roth-Schmidt-Nevanlinna bounds, or better, J. Number Theory 21 (1985), 347-389. MR 87f:11046

[St] W. Stoll, The Ahlfors-Weyl theory of meromorphic maps on parabolic manifolds, Lecture Notes in Mathematics 981, Springer-Verlag, 1983. MR 85c:32045

[Vi] A. Vitter, The lemma of the logarithmic derivative in several complex variables, Duke Math. J. 44 (1977), 89-104. MR 55:5903

[Vo] P. Vojta, Diophantine Approximations and Value Distribution Theory, Lecture Notes in Mathematics 1239, Springer-Verlag, 1987. MR 91k:11049

[Wo] P.M. Wong, On the second main theorem of Nevanlinna theory, Amer. J. Math. 111 (1989), 549-583. MR 91b:32030

[W-S] P.M. Wong and W. Stoll, Second main theorem of Nevanlinna theory for nonequidimensional meromorphic maps, Amer. J. Math. 116 (1994), 1031-1071. MR 95g:32042

[Ye 1] Z. Ye, On Nevanlinna's Error Terms, Duke Math. J. 64 (1991), 243-260. MR 93a:30039

[Ye 2] Z. Ye, On Nevanlinna's second main theorem in projective space, Invent. Math. 122 (1995), 475-507. MR 96j:32030

Department of Mathematics, University of Michigan, Ann Arbor, Michigan 48109

E-mail address: wcherry@math.1sa.umich.edu

Department of Mathematics, Northern Illinois University, DeKalb, Illinois 60115

E-mail address: ye@math.niu.edu 